\title{
MULTI-PHASE SIMULATION OF THE LIQUID COOLANT FLOW AROUND ROTATING CUTTING TOOL
}

\author{
L. Topinka ${ }^{1 *}$, M. Braeunig ${ }^{1}$, J. Regel ${ }^{1}$, M. Putz ${ }^{1,2}$, M. Dix ${ }^{1,2}$ \\ ${ }^{1}$ Chemnitz University of Technology, Institute for Machine Tools and Production Processes (IWP), Chemnitz, \\ Germany \\ ${ }^{2}$ Fraunhofer Institute for Machine Tools and Forming Technology IWU, Germany \\ *Corresponding author; e-mail: lukas.topinka@mb.tu-chemnitz.de
}

\begin{abstract}
The heat generated during the cutting processes significantly influences the manufacturing accuracy. For many machining tasks, the use of cooling lubricant is essential to achieve the necessary cooling and lubricating effect in the cutting zone. A reduction of a coolant supply due to resource-efficient or ecological efforts has an impact on the temperature field in the cutting tool and clamping system, which affects the thermal behavior of the frame structure and can cause its thermo-elastic deformations leading to machining inaccuracies. In this paper, the multi-phase CFD model for simulation of liquid coolant flow around the cutting tool was developed. The effect of rotation of the cutting tool causing turbulences was taken into account and the simulation of coolant flow was based in Eulerian approach as a continuous flow. Furthermore, this paper considers the modification of the simulation model for thermal simulations of the influence of coolant flow on the temperature field of the cutting tool and clamping system.
\end{abstract}

\section{Keywords:}

CFD-Simulation; Multi-phase simulation; Thermal investigation; Machine Tools; Tool cooling simulation

\section{INTRODUCTION}

The majority of materials used in metalworking machine tool components are negatively affected by temperature, both in terms of service life (e.g. cutting tool) and machining accuracy (e.g. structural deformation). The inaccuracy of machine tools due to thermal errors caused by heat generation in the cutting zone resulting from shear, friction and cutting energy is commonly known fact. In comparison with geometric, static and dynamically induced errors, the thermal error of the metal cutting machine tool may predominate [Braeunig 2018]. The use of cooling methods is necessary to ensure the thermal stability of the machining process. The current trend leads to use of dry machining [Putz 2018], which shows the possibility of reduction of temperature field in the cutting tool by up to $50 \%$ in comparison to machining without coolant [Perri 2016, Putz 2015, Braeunig 2019]. However, for many machining tasks is the use of liquid coolants still essential due to their high heat capacity ensuring greater efficiency of cooling effect. In addition, they also provide lubricating effect reducing the friction and adhesion between the tool and workpiece during the cutting operations, what thus leads to the lower heat generation [Helmig 2019].

Delivery of the liquid coolant into the cutting zone can be difficult, especially when high-speed machining. Because of high pressure occurring due to plastic deformation of materials during the cutting process, the coolant may never reach but only approach the cutting point [Liu 2021]. At high rotational speeds, the liquid may not even reach the inner zone of cutting tool teeth and the cooling process can be therefore less efficient. The use of numerical simulations can provide a better understanding of the cooling process leading to increase the cooling efficiency and decrease resource costs and the ecological impact on the environment [Neugebauer 2014].

In the previous studies [Perri 2016, Putz 2015] on the simulation of cutting tool cooling when dry machining, the use of single-phase simulation took place as the coolant and ambient air were the same fluid or phase. When using liquid coolant, the phenomenon of mixing two fluid of different phases occurs. Therefore, the use of single-phase simulations is no more possible and it is necessary to use the more complex multi-phase simulations.

The first step of a successful thermal simulation of multiple cooling strategies using the liquid coolants on the thermal regime of the cutting tool is to solve the flow behaviour of the coolant around rotating cutting tool. This paper thus presents a multi-phase simulation of liquid coolant flow around the cutting tool when conventional cooling with focus on a system consisting of the restricted area and machine components close around the cutting point. A numerical model developed in the environment of ANSYS CFX corresponds to a test stand used for the experimental investigations in order to verify numerical simulations. This 
simulation model approximates the flow of a liquid coolant around a rotating cutting tool in Eulerian approach with the possibility of a further extension of heat source on cutting edges of the tool tip. Within this, the simulation model will enable the evaluation of the efficiency of the cooling effect of a liquid coolant on the temperature field of a cutting tool under specific working conditions, which is further purpose of this simulation model.

\section{THE SIMULATION MODEL}

The numerical methods for computational fluid dynamics are highly demanding on calculation time and computational performance. As the machine tool is assembled of a large number of different components, the simulation model must focus only on the investigated area in order to obtain practicable calculation times [Braeunig 2019, Brier 2021]. In this case, the area of interest contains only components immediately nearby to the cutting point. The 3D model consists of a four blades milling tool with Taper ISO SK40, nozzle for coolant supply and restricted area of a surrounding air volume (see Fig. 1). The corresponding 3D models of components are simplified in order to shorten calculation times, but still accurate enough to keep simulation results reliable.

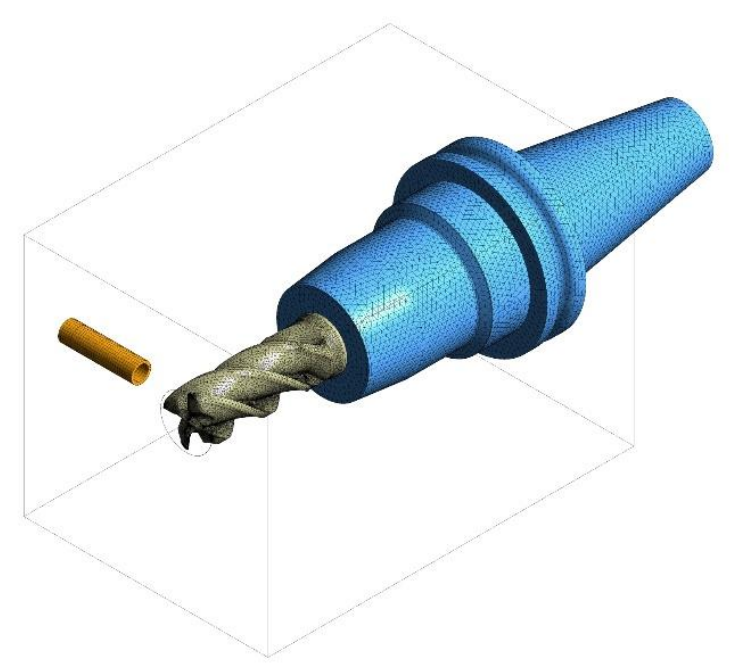

Fig. 1: $3 D C A D$ model of investigated area

\subsection{Meshing the 3D geometry}

The created $3 \mathrm{D}$ model can be set up for numerical simulations with the use of finite element method (FEM) only when the geometry is converted to mesh. The CFD simulation is very sensitive on the size of created mesh as the finer mesh would provide better environment for more precise calculation of flowing liquid, but on the other side, an increasing number of mesh elements also increases the calculation time and requirements for computational power. Therefore the mesh size is commonly adjusted during the creation and verification of the simulation model. After recording the coolants flow behavior (further described in section 3.1), the first simulation results were compared and the mesh size was adjusted on the basis of convergence of numerical calculation and nonsensible appearances in coolants flow, such as visibly damaged surface of the flowing coolant. Since the spiral geometry of the milling tool creates vortex flow air where occurs also the mixture with liquid phase of coolant, the mesh required strong refinement and the final mesh size was nearly $1,400,000$ elements.

\subsection{Definition of the model domains}

For numerical calculations of fluid dynamics, every part of the simulation model must be defined as a domain and connections between them are described as interfaces with appropriate physical properties. This simulation model consists of 6 domains and 8 interfaces. The schematic structure, which shows individual domains and their interfaces with arrow lines, is shown in the Fig. 2. Three domains filled with grey colour represent solid components of the investigated area: milling tool, tool chuck and coolant nozzle. Domains representing the milling tool and its chuck are set as rotational with speed corresponding to a rotational speed used in experimental investigations supporting the correct verification of simulation model. The influence of friction between the surface of these components and flowing liquid coolant, which can also have an impact on properties of fluids flow and its direction after the contact with a solid component, is also taken into account.

Other blue coloured domains are set as multi-phase fluid domains for representation of an ambient air volume around the solid components and flowing liquid coolant. In the definition of the simulation model, these ambient areas are named as Airvolumes. The Airvolume 1 domain represents the restricted area of workspace around the solid domains. Due to complexity of CFD simulations, the size of this ambient area is only covering the area nearby the cutting tool and its holder, but it is sufficient for the visualization of the coolant flow around these components. Although it is modelled as a restricted area, from the thermodynamic aspect it is defined as an open system, so the mass and heat exchanges are fully enabled with its surrounding environment. The definition of the physical properties of each of two phases, including the corresponding Surface Tension Coefficient between them, is based on the characteristics of both fluids used in supporting experimental investigations.

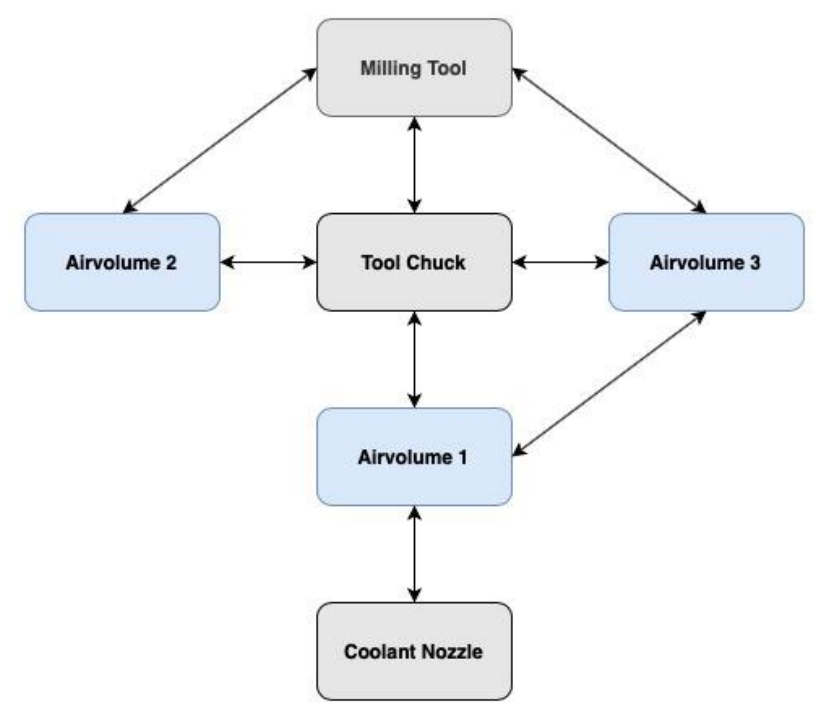

Fig. 2: Scheme of domains of the simulation model

Since the milling tool does not fill the complete hole in the clamping chuck, Airvolume 2 represents a small space between these two components. The Airvolume 3 does not represent another ambient space, which would not be possible to cover by previous two Airvolumes, but simplifies the simulation to shorten calculation time. In order to simulate the rotational movement of a component, it is only possible to rotate parts or domains, whose surfaces are parallel to the direction of movement. Otherwise, the mesh will be recalculated at every time-step of the simulation, 
called as transient mesh change, which greatly extend the computational time. However, the shape of the four blades milling tool does not meet this condition, as shown in red in Fig. 3 and thus causes the impossibility of its rotation without the transient mesh change. Therefore, a cylindrical domain was modelled, which fills the space of inner part of tool teeth and has a diameter of only $0,2 \mathrm{~mm}$ larger than the diameter of the tool, so its influence on a surrounding air volume is minimized. This enables the rotation of the tool without transient mesh change and therefore significantly shortens calculation time.

Because the two phases are mixed in these domains, a calculation approach for multi-phase simulations has to be specified. As mentioned above, due to high rotational speed of the cutting tool a significant concentration of fog particles or droplets dispersed into the environment can occur, but the numerical simulation of flowing droplets is highly demanding on computational power.

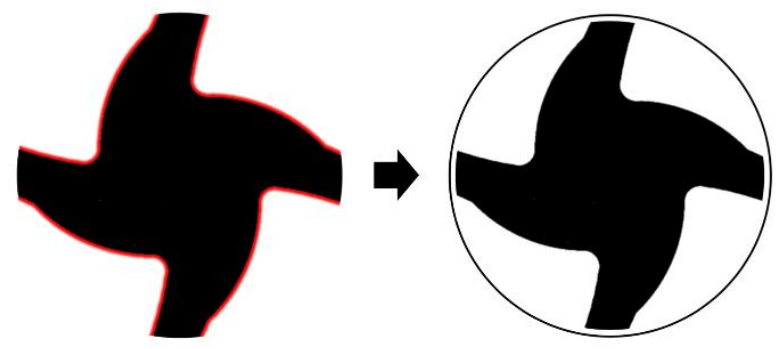

Fig. 3: Scheme of Airvolume 3

However, the further purpose of this simulation model is to simulate the influence of the liquid coolant flow on the thermal regime of the cutting tool and its holder and not on the environment or other components of the machine tool. Therefore, there is no need to simulate the trajectories of these droplets and thus this simulation model is set up with the Eulerian approach, which simulates the continuous liquid flow, but do not visualise the droplets and thereby assures significant shorter calculation times.

Furthermore, the simulation must consider all the physical properties influencing the coolant flow behavior and thus the gravitational force causing the curvature of the liquid coolant flow was also taken into account. This was assured by the applying of the function Buoyancy in the simulation model.

\section{LIQUID COOLANT FLOW}

The nature of the CFD simulations, especially when a mixture of multiple thermodynamic phases occurs, is a complex field of simulations itself. The numerical calculation of the flow of liquid is based on the Navier-Stokes equations and requires proper definition of the boundary conditions.

However, solving these equations alone is only possible when laminar flow. In this case, due to high rotational speeds, the spiral geometry of the milling tool and the phenomenon of the mixing multiple thermodynamic phases, there occurs a turbulent behaviour of the flow, which requires the extension of these fundamental NavierStokes equations of mathematical turbulence models, which will be mentioned later in the section 3.2. To be able to validate the simulation model, the numerical simulations must be verified with supporting experimental investigations. For both the simulations and experiments, example cases with varying parameters were selected. In order to simplify the investigation due to the complexity of CFD calculations, there were only two parameters changing - the rotational speed of the tool (RPM) and the speed or volume of coolant supply. The series of chosen RPMs was following: $500,1000,3000,6000 \mathrm{~min}^{-1}$. All of these RPMs were combined with two flow rates of coolant supply: 2 and $5,5 \mathrm{l} / \mathrm{min}$

\subsection{Experimental investigations}

The experimental investigation of coolant flow was provided on a test stand with components, on which basis the simulation model was developed. The aim of these experimental investigations was to record the behaviour of the coolant flow around rotating cutting tool in order to find a proper setting of the flow behaviour of the simulation model. The evaluation of results of the experimental investigations was provided according to optical aspects, as any measurements of coolant behaviour (such as measurements of distribution of fluid particles into the air space when dispersed flows) could not provide as valuable data for this kind of numerical simulations as visual recording with a camera.

When the flowing coolant comes into contact with a rotating tool, especially at higher speeds, part of a continuous coolant flow changes its behaviour to a field of droplets distributed into the surrounding air space. To record these flowing droplets, a high-speed camera would be necessary and a numerical simulation of dispersed particles would place very high demands on computing power. As already mentioned above, the further purpose of this simulation model lies particularly in thermal simulations as the focus of this research is to investigate the influence of the liquid coolant on the temperature field of the cutting tool. Therefore, also by supporting experimental investigations, there was no need to track all dispersed droplets, but mainly focus on the behaviour of continuous flow of the liquid coolant around the tool.

Hence the use of ordinary camera was completely sufficient to provide necessary recordings of the coolant flow. Since the machine workspace must be closed when the milling tool is rotating, the camera was placed into the working area. Each coolant flow scenario was thereby recorded from two positions - a frontal view at the tool tip and from above the tool to monitor the flow on the plane along the tool axis. Exemplary results from experimental

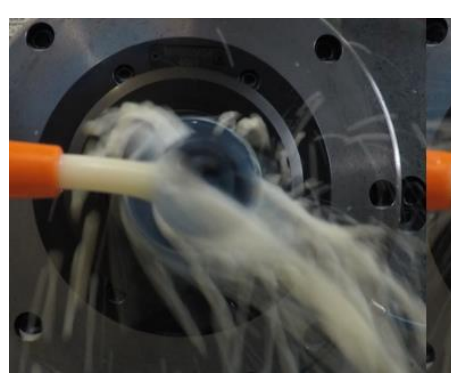

(a) $500 \mathrm{rpm}$

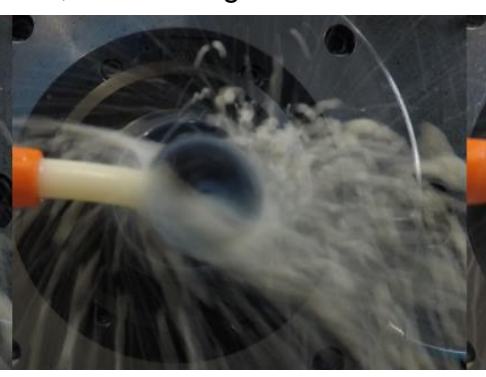

(b) $1000 \mathrm{rpm}$

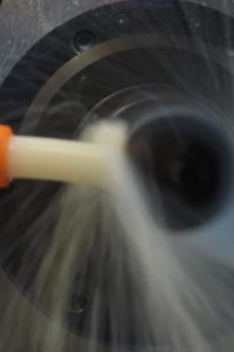

(c) $3000 \mathrm{rpm}$

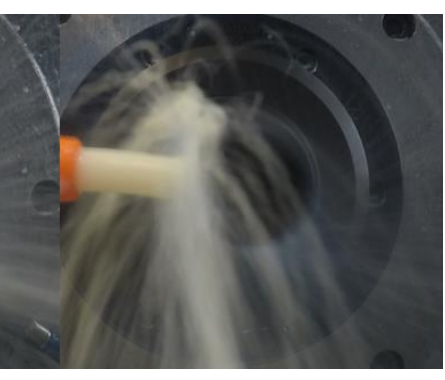

(d) $6000 \mathrm{rpm}$

Fig. 4: Coolant flow around rotating cutting tool

MM Science Journal | 2021 | NOVEMBER - Special Issue on HSM2021 
investigations showing the differences of coolant flow around the milling tool at different rotational speeds are given in Fig. 4.

\subsection{Evaluation of experimental results and comparison with numerical simulations}

After recording the flow behaviour from supporting experiments, it was necessary to define the method, how these results will be evaluated and compared with the simulation results. The simplification of the simulation model also had to be taken into account, since the goal was to simulate the flow behaviour of coolant around rotating milling tool with certain similarity practice-acceptable time durations. This would further enable the simulation under real cutting conditions. Therefore, the evaluation was provided without quantitative methods of measurements, since there was no variable (such as speed or volume), which would be easy to measure, but only according to qualitative optical aspects. Thus it was necessary to provide a definition of evaluation method to distinguish between different flow behaviour. The evaluation of experimental results and its comparison with the results from numerical simulations are provided in the Fig. 5, where the different flow behaviours are marked with colours for better understanding of the methodology.

The statements of evaluation method are following:

- if the density of flowing dispersed fluid particles enables visibility of background behind this flow (e.g. spindle housing), this area was called as droplet field (green colour)

- if the coolant flow is continuous or the density of droplets is so high, that the background is not sufficiently recognisable, this was called continuous coolant flow (red colour)

The rendering of results from numerical CFD simulations was provided through two functions. First function Isosurface, visualize the boundary layer between the air and liquid phase. However, it does not show particles smaller than the dimension of mesh. This can be influenced by changing the dimension of mesh, but it would also have a significant influence on calculation time. The second used function, Volume Rendering, enables to visualize the percentage ratio of both phases in areas, where the dispersed fluid particles flow. Thus it is possible to compare the dispersion with results from experimental investigations while keeping the calculation times in practice-acceptable time durations.

The key focus of the CFD simulation was on the continuous coolant flow (marked with red colour), since this is a part of coolant, which provides a significant cooling effect and thus influences the temperature field of the tool. The area of droplet field (marked with green colour) provides rather the cooling effect on the other components of the machine during real milling processes or can also have an influence on lowering the friction between the tool and workpiece, which would lower generated heat. Increasing the precision of this field would require much finer meshing and thus place higher demands on computational power. However, this was not an aim of this simulation model and therefore it was not necessary to focus on receiving high precision in this field.

Once the simulation results showed the similar coolants behavior containing the division of the coolants flow from the nozzle into continuous coolant flow and dispersed droplets after the contact with rotating tool, the simulation results could be declared as sufficiently acceptable. The amount and the direction of the main continuous coolant flow could not differ significantly (within the range of $10^{\circ}$

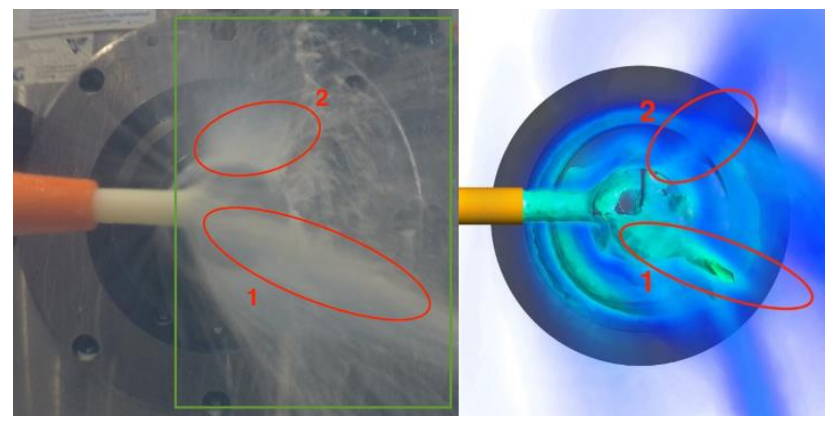

Fig. 5: Comparison of the experimental and simulation results (5,5 I/min; $500 \mathrm{rpm})$

difference) in comparison to recorded coolants flow behavior during the experiments on the test stand.

\subsection{Turbulence model}

As mentioned at the beginning of section 3, the numerical calculation of the fluid flow behaviour on the basis of fundamental differential Navier-Stokes equations is only possible when the flow is laminar or does not include turbulent behaviour. However, the fluid behaviour around rotating end mill tool is more complex. The spiral shaped design of cutting edges generate the vortex flow of an ambient air. Then, when the liquid coolant supplies, the flow of the coolant with much higher density rapidly disturbs the surrounding airflow and the phenomenon of the mixing of these two phases occurs. This is accompanied by the formation of turbulence in this area. Therefore, the numerical calculations must be extended by mathematical turbulence models. The most commonly used turbulence models in CFD simulations are k-epsilon (k- $\varepsilon$ ), k-omega (k$\omega)$ and Shear Stress Transport (SST) [Lecheler 2018]. In order to find the most suitable turbulence model, the chosen example cases were simulated with all these turbulence models and results compared with experimental investigations.

\section{$k$ - $\varepsilon$-Model}

The results of simulations with the first mentioned turbulence model, $k-\varepsilon$ (Fig. 6b), show, that this model provides a satisfactory representation of the coolant flow around the cutting edges as well as the adherence of the coolant around the tool holder. On the other side, the coolant flow after the contact with the tool was not adequately calculated. The intensity and direction deviate significantly from the measurements as the course decrease more steeply than in experimental investigations. Also, the area of flowing dispersed droplets was not shown. $k$ - $\omega$-Model

The second used turbulence model k- $\omega$ (Fig. 6c) shows some improvements in comparison to previous $\mathrm{k}-\varepsilon$-Model. The representation of the coolant around the milling tool was similar and the adhesion of the coolant in the area of the tool holder is slightly more obvious. The coolant flow after the contact with the tool had higher intensity and the direction did not deviate as much as in previous model. There were also improvements in the calculation of the area of flowing droplets.

\section{SST-Model}

The last investigated turbulence model was SST (Fig. 6d). These results are closest to the real coolant flow behaviour around rotating milling tool and tool holder during experimental investigations. The continuous coolant flow also corresponds to the experimental investigation in both intensity and course. Even all of the simulation cases were provided in Eulerian approach, this turbulence model shows 
a good visualization of dispersed areas. Compared to the other two turbulence models, this model shows the highest agreement of results regarding the recordings of results from experimental investigations. Also, calculations with all selected turbulent models did not differ much in calculation time. Therefore, the SST turbulence model was selected as the most suitable for the developed simulation model and was used in further simulations of coolant flow. The Fig. 5 illustrates a comparison of example results of numerical simulations for each turbulence model with recording from experimental investigation when rotational speed of 500 rpm and coolant supply $5,5 \mathrm{I} / \mathrm{min}$.

\subsection{Full CFD simulations}

After the selection of turbulence model, the simulation model was prepared for simulations of the various cooling scenarios, which were described in the section 3 . These simulations were performed in the ANSYS CFX R19.1 environment on a computer with a quad-core Intel Xeon(R) 3.3 GHz processor. Since the simulations were run transient due to the high computational complexity of the cases, it was necessary to determine a time interval after which the simulated flow partially stabilized compared to the initial state when the simulation was started. It was found that the stabilization of the flow after the initial impact of the fluid on the rotating tool occurred after approximately 2-3 seconds. As this was only a simulation of this flow, an interval of 5 seconds was set to ensure sufficient time for the more challenging of the cases. The simulation of this interval with a timestep of 0.01 seconds took almost 6 hours.

The simulation results of all of the investigated scenarios with SST turbulence model have shown a good accordance with most of the results from experimental investigations. However, due to turbulences and instabilities of the numerical calculations, under some conditions it was difficult to predict the behavior and direction of coolant flow after the contact with rotating tool, such as the field of droplets. Especially when higher rotational speeds, these instabilities were more recognizable. As seen for example in Fig. 4, when higher rotational speed, the greater part of coolant flow changes its behaviour from continuous flow into droplet field. Since the calculations were provided in Eulerian approach, which is not primarily intended for the
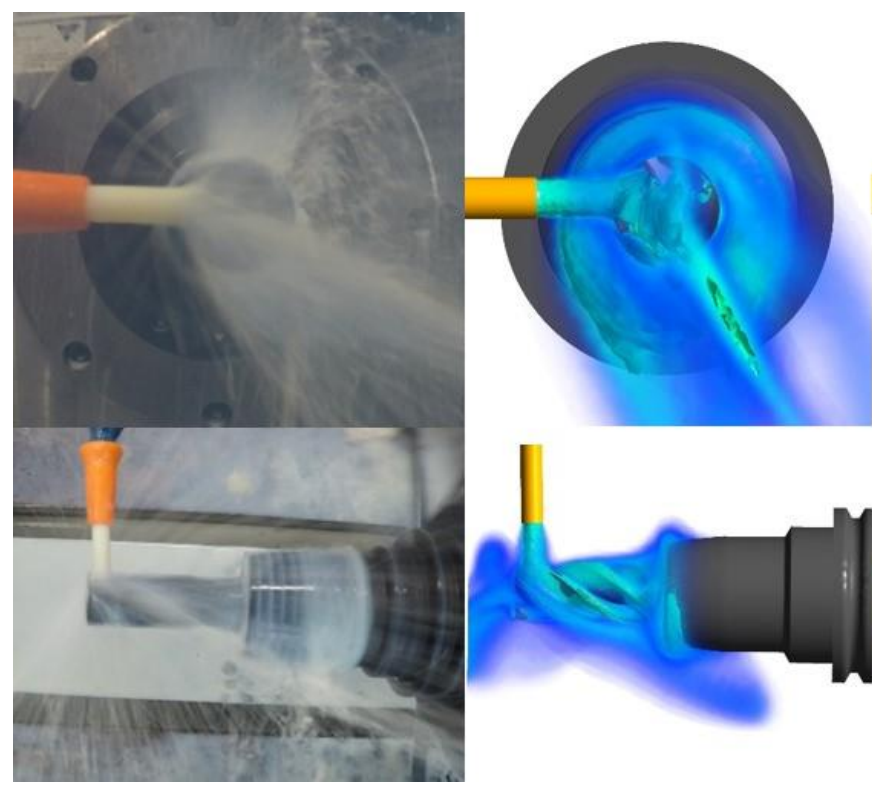

(a) Experiment simulations of dispersed particles, some inaccuracies in the results appeared. This could not only be caused by the settings for the continuous flow simulations, but also the mesh and timestep settings of the simulation or the fact that a given type of multiphase flow is often considered as too challenging for the solution of the Navier-Stokes equations themselves. When setting the mesh or the timestep, it was necessary to find the appropriate settings both for sufficient quality of the computed result and for keeping the simulation time as short as possible. Thus the results of simulation cannot fully correspond with experimental investigations.

This can be improved, for example, with shorter timestep in transient calculations or by selecting another calculation approach, such as Lagrangian, which is used for simulations of dispersed particles, but it would also rapidly extend simulation time. However, the similarity between simulated and experimentally recorded results is already at a sufficient level to allow further application of this simulation model for thermal simulations under realistic cutting conditions.

\section{SUMMARY AND OUTLOOK}

Simulation of the coolant flow around rotating milling tool with a spiral shaped design of the cutting edges consider only small area on a real-world scale, but reveals certain difficulties in the aspect of numerical CFD simulations. These difficulties are caused mostly by the phenomenon of the mixing of two fluid phases with different densities in a vortex area, which leads to the effect of turbulence, and dispersion of liquid into the environment as a function of rotational speed of the cutting tool. This phenomenon places high demands on the definition of the mesh, since not only the flow itself is calculated, but in the aspect of numerical calculations, the exchange of the variables between several domains is also contained in this area.

Taking into account the simulation of small particles or droplets of liquid spreading into the environment is difficult and increases demands on computational power and accuracy of the simulation itself. However, the numerical simulations in Eulerian approach with SST turbulence model showed, that it is still possible to simulate the liquid

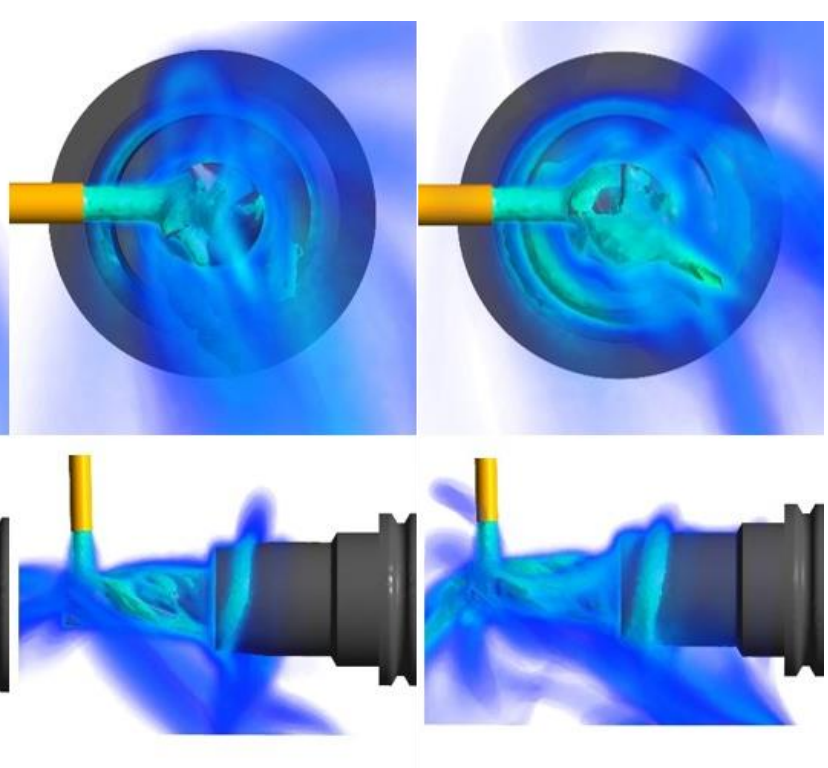

(c) $k-\omega-M o d e l$

Fig. 6: Comparison of different turbulence models with real flow behaviour

MM Science Journal | 2021 | NOVEMBER - Special Issue on HSM2021 
coolant flow around a rotational cutting tool and spreading droplets field with certain precision required for further simulation of cooling effects under realistic working conditions. The results still contain some inaccuracies caused mostly due to difficulties when calculating dispersion and turbulent behaviour around cutting edges. However, it is possible to simplify the conditions in order to shorten calculation times into practice-acceptable durations and still simulate the coolant flow with certain similarity to experimentally recorded real conditions.

The results of these simulations thus show the possibility of future development of this simulation model to allow thermal simulations of the cooling effects of multiple cooling strategies. This would lead to a more efficient use of coolants, thereby reducing resource costs and ecological impact on the environment. In addition to a more efficient use of coolants, the calculation of the TCP displacement would lead to higher machining accuracy.

\subsection{Outlook on thermal simulation}

As already mentioned in this article, the further purpose of this simulation model is to simulate the influence of the liquid coolant flow on the thermal regime of a cutting too and its holder. After obtaining a multiphase simulation of the coolant flow with satisfactory results, the simulation model can be extended by the parameters and settings required for thermal simulations.

However, first attempts to evaluate the interaction between the milling tool or its surface and the flowing coolant have shown complications and erroneous behaviour of this interface. The visualisation of the coolant flow velocity on the tool surface or the pressure caused by their contact was limited and showed unrealistic values, usually at much lower scales than should be present. This is also expected to have a negative effect on the calculation of heat transfer between the tool and the coolant, and thus on the simulation of the cooling effect itself. This erroneous behaviour in the interaction between the coolant and the milling tool has revealed another challenge and the need for further simulation model development in this area to ensure full functionality of further thermal simulations under realistic cutting conditions.

Once the erroneous behaviour at the given interface is debugged, the materials and thermodynamic properties of each domain can be defined based on the components used on the test stand supporting the verification of the simulation model. In addition, the heat transfer mechanisms between the domains as well as at the tool holder cone where the heat is transferred to/from the machine spindle need to be specified. However, the simulation of a realistic cutting process with chip formation would impose very high computational power requirements. To simplify heat generation in both simulation and supporting verification experiments, the tool tip can be heated by an induction device and the corresponding heat flux added in the simulation model. This allows to simulate simplified conditions of heat generation and transfer through the components and to compare the effectiveness of the cooling methods used on the temperature field of the given components.

Furthermore, after the establishment of the fully functional thermal simulation model, the goal is to create knowledge of the influence of the coolant on the thermal regime of cutting components close to the cutting zone under various cooling scenarios and cutting conditions. With this knowledge, it will be possible to create methodology for the development of the real-time correction method of thermally induced errors and TCP displacements. This correction system can be implemented into the machine control system and in the combination with temperature sensors placed on the tool holder or the spindle nose, the correction of TCP displacement can be automatically calculated and actively controlled during the machining process. This, in combination with active control of the coolant supply, would rapidly increase the precision of machining with higher efficiency of the application of coolants and thus lower resource costs and ecological impact on the environment.

\section{ACKNOWLEDGEMENTS}

The work is funded by the German Research Foundation Project-ID 174223256 - TRR96. Authors thank for the support.

\section{REFERENCES}

[Braeunig 2018] Braeunig, M., Regel, J., Richter, C. and Putz, M. Industrial relevance and causes of thermal issues in machine tools. In: $1^{\text {st }}$ Conference on Thermal Issues in Machine Tools, Dresden, 2018

[Braeunig 2019] Braeunig, M., Regel, J., Glaenzel, J. and Putz, M. Effects of cooling lubricant on the thermal regime in the working space of machine tools. Procedia Manufacturing, 2019, pp 327-334, DOI: 10.1016/j.promfg.2019.04.040

[Brier 2021] Brier, S., Regel, J., Putz, M. and Dix, M. Unidirectional coupled finite element simulation of thermoelastic TCP-displacement through milling process caused heat load. In: $2^{\text {nd }}$ International Conference on Thermal Issues in Machine Tools, Prag, 2021

[Helmig 2019] Helmig, T., Peng, B., Ehrenpreis, C., Augspurger, T., Frekers, Y., Kneer, R. and Bergs, T. A Coupling Approach Combining Computational Fluid Dynamics and Finite Element Method to Predict Cutting Fluid Effects on the Tool Temperature in Cutting Processes. Journal of Manufacturing Science and Engineering, 2019, Vol.141, DOI: 10.1115/1.4044102

[Lecheler 2018] Lecheler, S. Numerische Strömungsberechnung. Wiesbaden: Springer Vieweg, pp 200, ISBN 978-3-658-19191-7

[Liu 2021] Liu, H., Helmig, T., Augspurger, T., Nhat, N., Kneer, R. and Bergs, T. Modeling the cooling effect of the cutting fluid in machining using a coupled FE-CFD simulation. In: $2^{\text {nd }}$ International Conference on Thermal Issues in Machine Tools, Prag, 2021

[Neugebauer 2014] Neugebauer, R., Ihlenfeld, S., Zwingenberger, C., Glänzel, J. and Richter, C. Modelling of Thermal Interaction between Environment and Machine Tool. Thermo-energetic design of machine tools, Berlin, 2014, pp 111-124

[Perri 2016] Perri, G.M., Bräunig, M., Gironimo, G.D., Putz, M., Tarallo, A. and Wittstock, V. Numerical modelling and analysis of the influence of an air cooling system on a milling machine in virtual environment. The International Journal of Advanced Manufacturing Technology, 2016, Vol.86, pp 1853-1864

[Putz 2015] Putz, M., Wittstock, V., Semmler, U. and Bräunig, M. Simulation-based thermal investigation of the cutting tool in the environment of single-phase fluxes. The International Journal of Advanced Manufacturing Technology, 2015, Vol.83, pp 117-121

[Putz 2018] Putz, M., Richter, C., Regel, J. and Bräunig, M. Industrial consideration of thermal issues on machine tools. Production Engineering - Research and Development, 2018, Vol.12, pp 723-736 